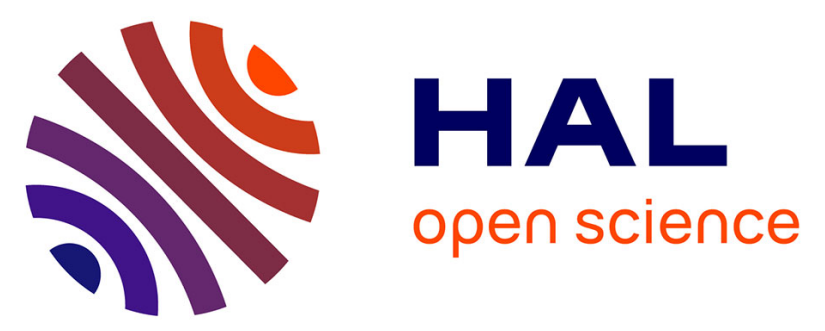

\title{
Delay Estimation and Predictive Control of Uncertain Systems With Input Delay: Application to a DC Motor
}

Vincent Léchappé, A Gonzáles, Franck Plestan, Jesus de Leon, Emmanuel Moulay, Alain Glumineau, Sébastien Rouquet

\section{- To cite this version:}

Vincent Léchappé, A Gonzáles, Franck Plestan, Jesus de Leon, Emmanuel Moulay, et al.. Delay Estimation and Predictive Control of Uncertain Systems With Input Delay: Application to a DC Motor. IEEE Transactions on Industrial Electronics, 2016, 63 (9), pp.5849 - 5857. 10.1109/TIE.2016.2527692 . hal-01273984

\section{HAL Id: hal-01273984 \\ https://hal.science/hal-01273984}

Submitted on 15 Feb 2016

HAL is a multi-disciplinary open access archive for the deposit and dissemination of scientific research documents, whether they are published or not. The documents may come from teaching and research institutions in France or abroad, or from public or private research centers.
L'archive ouverte pluridisciplinaire HAL, est destinée au dépôt et à la diffusion de documents scientifiques de niveau recherche, publiés ou non, émanant des établissements d'enseignement et de recherche français ou étrangers, des laboratoires publics ou privés. 


\title{
Delay Estimation and Predictive Control of Uncertain Systems With Input Delay: Application to a DC Motor
}

\author{
V. Léchappé, S. Rouquet, A. Gonzáles, F. Plestan, J. De León, E. Moulay and A. Glumineau
}

\begin{abstract}
It is well-known that standard predictive techniques are not very robust to parameter uncertainties and to external disturbances. Furthermore, they require the exact knowledge of the delay. In practice, these constraints are rarely satisfied. In this paper, solutions are presented to allow the use of predictive control in presence of external disturbances, parameter uncertainties and an unknown input delay. First, a recent predictive control method developed to attenuate the effect of external disturbances is shown to be also robust to parameter uncertainties. In addition, a delay estimator is presented to estimate unknown time-varying delays. Theoretical results are widely illustrated through experimental tests on a DC motor.
\end{abstract}

Index Terms-Delay estimation, predictive control, input delay.

\section{INTRODUCTION}

On the one hand, input delays can appear because of the physical nature of the plant: they are usually due to transport phenomenon. On the other hand, input delays can arise from communication latencies, computation time or sensor measurements: in that case, they are extrinsic to the system. The latter are more and more common because of the fast development of remote controllers and control over networks (see [1] for further applications).

The standard approach to control such systems is the wellknown Smith predictor introduced in [2]. Then, the finite spectrum assignment technique and the Artstein reduction method respectively in [3] and [4] have extended Smith's result. However, these methods have a major problem: they are not very robust to external disturbances and modeling errors. In addition, the exact value of the delay is required to compute the prediction which is very sensitive to delay mismatch [5]. In order to improve the problem of disturbance attenuation, a new predictive scheme has been introduced in [6]. This solution offers new perspectives for the control of uncertain input delay systems. In particular, it can be applied for the control of electrical machines.

DC motors are commonly used in many areas such as robotics or industry. Furthermore, the simple modeling facilitates its use as a benchmark system for the evaluation of new control laws. Numerous techniques have been applied for driving DC motors [7]. However, they do not consider any delay. This issue is studied in this paper.

Among the few existing works concerning DC motors with delayed inputs, one can cite [8] which shows the influence of the delay time distribution on the stability of a DC motor with a PI controller. In [9], an observer-based delay compensator is designed in the discrete framework associated with buffers to reduce the unknown delay variations. An adaptive controller is used in [10] to follow the "Quality-of-Service" (QoS) variations of the network. These works consider very small delays (less than the sampling period) and a perfect model (no parameter uncertainty, no external disturbance). The control of a DC motor with a large delay and external constant disturbances is achieved in [11] by using the recent method of [6].

All these works deal with constant and known delays. However, if the control is carried out over a network (networked control system), the delay is usually time-varying. Recently, numerous works on the control of DC motor through communication networks have been published. They generally take into account random delays as in [12] and often also both delay and packet dropout as in [13], [14], [15], [16]. Memoryless controllers are used in [13] and [14] because the delays are small (less than $100 \mathrm{~ms}$ ). When the delay becomes larger, predictive techniques are used as in [12] and [15]. The work [16] does not use predictive control, that is why the performances are clearly degraded when the delay increases. Among the works that use predictive techniques, none considers an external disturbance or parameter uncertainties.

In most of the cases, the delay is not available for measurement. Therefore, it is useful to have delay estimators in order to approximate the value of the delay. Various methods are available but they are generally run offline or they deal only with constant delays. In addition, most of the works only deal with "open-loop" estimation. That is to say that they estimate the delay but do not use the estimated value to design a controller [17], [18]. However, few works combined predictive control with delay estimation. As far as the authors know, only four results are reported in the literature: [19], [20], [21] [22]. In [19] and [20], the delay is constant and the algorithm is very complex to implement. The work in [21] deals with transfer function systems and does not consider parameter uncertainties nor external disturbance. In [22], the delay estimation technique is based on a particular structure of the model and cannot be used for the DC motor.

One of the contribution of this work is to extend the result of [6] to systems with parameter uncertainties. Besides, a delay estimator is designed for unknown time-varying delays. Finally, the combination of the predictive method with the delay estimator is tested experimentally on a DC motor with a large input delay and unmodeled dynamics. An accurate speed tracking is achieved in presence of a large unknown delay, 
external disturbance and parameter uncertainties.

The article is organized as follows. In Section II, the predictive scheme of [6] is recalled and an analysis of its robustness to parameter uncertainties is worked out. A delay estimator is proposed in Section III and a convergence proof is given for unknown time-varying delays. The DC motor model and the experimental setup are introduced in Section IV-A. Experimental results are shown in Section IV-B. Finally, the conclusion and some future developments are outlined in Section V.

\section{PREDiCTIVE CONTROL OF UNCERTAIN SYSTEMS WITH KNOWN AND CONSTANT DELAY}

The results presented thereafter are an extension of [6] to systems with parameter uncertainties. The systems under consideration are LTI systems with uncertainties $\Delta A, \Delta B$ and an additive external perturbation $d$ :

$$
\dot{x}(t)=(A+\Delta A) x(t)+(B+\Delta B) u(t-h)+d
$$

with $x(t) \in \mathbb{R}^{n}, u(t) \in \mathbb{R}^{m}, d(t) \in \mathbb{R}^{n}, A \in \mathbb{R}^{n \times n}, B \in$ $\mathbb{R}^{n \times m}$ and the constant and known delay $h \in \mathbb{R}$. The following assumptions are made:

Assumption 1: The matrices $A$ and $B$ are known and $(A, B)$ is controllable.

Assumption 2: The state $x(t)$ is measurable.

Assumption 3: The disturbance $d$ is piecewise constant ${ }^{1}$ and unknown.

Assumption 4: The matrices $\Delta A$ and $\Delta B$ are constant and unknown and do not affect the controlability property.

In addition, it is assumed that the reference $x_{r}$ is constant and verifies:

$$
0=A x_{r}+B u_{r}
$$

where $u_{r}$ is the constant control input required to maintain $x$ at equilibrium point $x_{r}$. Equation (2) allows to compute a controller of the form $u(t)=u^{\prime}(t)+u_{r}$ then the tracking error $e(t)=x(t)-x_{r}$ verifies:

$$
\begin{aligned}
\dot{e}(t) & =A e(t)+B u^{\prime}(t)+A x_{r}+B u_{r} \\
& =A e(t)+B u^{\prime}(t) .
\end{aligned}
$$

The Finite Spectrum Assignment (FSA) technique in [3] is based on the following prediction

$$
x_{\hat{p}}(t)=e^{A h} x(t)+\int_{t-h}^{t} e^{A(t-s)} B u(s) \mathrm{d} s
$$

for all $t \geq 0$. The term $x_{\hat{p}}$ will be called the "standard prediction" in the sequel because it is the most widely used prediction in the literature of input delay systems [4], [23]. The state prediction (4) allows to turn (1) into the system

$$
\dot{x}_{\hat{p}}(t)=(A+\Delta A) x_{\hat{p}}(t)+B u(t)+\phi_{1}(t),
$$

\footnotetext{
${ }^{1}$ This condition is imposed by an experimental constraint (Remark 3) but similar results would hold if the disturbance is sufficiently slow-varying with respect to the delay size [6].
}

with

$\phi_{1}(t)=e^{A h} d+e^{A h} \Delta B u(t-h)-\Delta A \int_{t-h}^{t} e^{A(t-s)} B u(s) \mathrm{d} s$.

The term $\phi_{1}$ can be seen as a perturbation of the delay-free system

$$
\dot{x}_{\hat{p}}(t)=(A+\Delta A) x_{\hat{p}}(t)+B u(t) .
$$

This transformation is very close to the Artstein reduction [4] where the substitution is carried out with $z(t)=e^{-A h} x_{\hat{p}}(t)$. Since (7) is not retarded anymore, all the controllers for delayfree systems can be used to stabilize it around $x_{r}$. In particular, PID or sliding mode algorithms will be necessary to reject the perturbation $\phi_{1}$ and make $x_{\hat{p}}(t)$ tend to $x_{r}$. If $x_{\hat{p}}(t)$ tends to $x_{r}$, it is expected that $x(t)$ tends to $x_{r}$. However, $x_{\hat{p}}$ is not the exact prediction because it does not take into account the perturbation; so there is an unavoidable error when $d \neq 0$, $\Delta A \neq 0$ or $\Delta B \neq 0$. The consequence of this error is stated in the next proposition where $\tilde{x}=x_{\hat{p}}-x_{r}$ :

Proposition 1: Consider system (1) satisfying Assumptions 1-4. Suppose that there exists a predictive controller $u(\tilde{x})$ continuous at the origin such that $\tilde{x}(t)$ and its time-derivative tend to zero. Then, the convergence of the state $x(t)$ to the reference $x_{r}$ is not ensured.

Proof: The dynamics of $\tilde{x}$ is governed by the equation

$$
\dot{\tilde{x}}(t)=(A+\Delta A) \tilde{x}(t)+\Delta A x_{r}-B u_{r}+B u(t)+\phi_{1}(t) .
$$

Assuming that there exists a controller continuous at the origin $^{2}$ such that $\tilde{x}$ converges to zero, then, one has $\lim _{t \rightarrow+\infty} u(\tilde{x}(t))=u\left(\lim _{t \rightarrow+\infty} \tilde{x}(t)\right)=u(\tilde{x}=0)=u_{\infty}$. Passing to the limit in (8), it can be deduced that

$$
B u_{\infty}=B u_{r}+\Phi
$$

with $\Phi=-\Delta A x_{r}-e^{A h} \Delta B u_{\infty}-e^{A h} d+\Delta A \int_{-h}^{0} e^{-A s} \mathrm{~d} s B u_{\infty}$. Reminding that $A x_{r}=-B u_{r}$, (9) becomes

$$
B u_{\infty}=-A x_{r}+\Phi .
$$

Besides, from (4), it follows that

$$
x_{r}=e^{A h} x_{\infty}+\int_{-h}^{0} e^{-A s} \mathrm{~d} s B u_{\infty}
$$

with $\lim _{t \rightarrow+\infty} x(t)=x_{\infty}$. Combining (10) and (11) and noting that $A$ and $e^{-A s}$ commute and that $\int_{-h}^{0} A e^{-A s} \mathrm{~d} s=I_{n}-e^{A h}$, one gets

$$
0=e^{A h}\left(x_{\infty}-x_{r}\right)+\int_{-h}^{0} e^{-A s} \mathrm{~d} s \Phi
$$

Since $-\int_{-h}^{0} e^{-A s} \mathrm{~d} s \Phi \neq 0$ for all $\Delta A$ and $\Delta B$, we obtain $x_{\infty} \neq x_{r}$.

${ }^{2}$ If $u$ is not continuous at the origin but one has $\tilde{x}(t)=0$ for all $t>T$, then it follows that $u(\tilde{x}(t))=u(\tilde{x}=0)$ for all $t>T$. 
Notice that when the model is perfectly known $(\Phi=0)$ then $x_{\infty}=x_{r}$ but when there are some unmodeled dynamics it is not possible to drive $x$ to $x_{r}$ with the standard prediction. Therefore, a new state prediction has been proposed:

Definition 1 ([6]): The new prediction is defined by

$$
X_{\hat{p}}(t)=x_{\hat{p}}(t)+x(t)-x_{\hat{p}}(t-h)
$$

for all $t \geq h$, with $x_{\hat{p}}$ given by (4).

Remark 1: $X_{\hat{p}}$ can be computed without any knowledge of $d$ but it requires the knowledge of $h$.

Similarly to (5), system (1) can be turned into

$$
\dot{X}_{\hat{p}}(t)=(A+\Delta A) X_{\hat{p}}(t)+B u(t)+\phi_{2}(t)
$$

with

$$
\begin{aligned}
\phi_{2}(t)= & d+\Delta B u(t-h) \\
& -\Delta A \int_{t-h}^{t} e^{A(t-s)} B[u(s)-u(s-h)] \mathrm{d} s \\
& +e^{A h} \Delta B[u(t-h)-u(t-2 h)] .
\end{aligned}
$$

The term $\phi_{2}$ can be seen as a perturbation of the delay-free system

$$
\dot{X}_{\hat{p}}(t)=(A+\Delta A) X_{\hat{p}}(t)+B u(t) .
$$

Then one has the following result where $\tilde{X}=X_{\hat{p}}-x_{r}$.

Proposition 2: Consider system (1) satisfying Assumptions 1-4. Suppose that there exists a predictive controller $u(\tilde{X})$ continuous at the origin such that $\tilde{X}(t)$ and its time-derivative tend to zero. Then, the state $x(t)$ converges to the reference $x_{r}$. equation

Proof: The tracking error dynamics $\tilde{X}$ is governed by the

$$
\dot{\tilde{X}}(t)=(A+\Delta A) \tilde{X}(t)+\Delta A x_{r}-B u_{r}+B u(t)+\phi_{2}(t) .
$$

Assuming that there exists a controller continuous at the origin such that $\tilde{X}$ converges to zero, it can be deduced from (17) that

$$
(B+\Delta B) u_{\infty}=B u_{r}-\Delta A x_{r}-d
$$

where $\lim _{t \rightarrow+\infty} u(t)=u_{\infty}$ (see the proof of Proposition 1 for the existence of the limit). Reminding that $A x_{r}=-B u_{r}$, (18) becomes

$$
(B+\Delta B) u_{\infty}=-(A+\Delta A) x_{r}-d .
$$

Besides, from (13), it follows that

$$
\begin{aligned}
X_{\hat{p}}(t)= & e^{A h}[x(t)-x(t-h)]+e^{(A+\Delta A) h} x(t-h) \\
& +\int_{t-h}^{t} e^{A(t-s)} B[u(s)-u(s-h)] \mathrm{d} s \\
& +\int_{-h}^{0} e^{-(A+\Delta A) s}[(B+\Delta B) u(t+s-h)+d] \mathrm{d} s
\end{aligned}
$$

so if $\lim _{t \rightarrow+\infty} \tilde{X}(t)=0$, one has

$$
x_{r}=e^{(A+\Delta A) h} x_{\infty}+\int_{-h}^{0} e^{-(A+\Delta A) s} \mathrm{~d} s\left[(B+\Delta B) u_{\infty}+d\right]
$$

where $\lim _{t \rightarrow+\infty} x(t)=x_{\infty}$. Combining (19) and (21) and noting that $A+\Delta A$ and $e^{-(A+\Delta A) s}$ commute and that $-\int_{-h}^{0}(A+$ $\Delta A) e^{-(A+\Delta A) s} \mathrm{~d} s=I_{n}-e^{(A+\Delta A) h}$ leads to

$$
0=e^{(A+\Delta A) h}\left[x_{\infty}-x_{r}\right] .
$$

Since $e^{(A+\Delta A) h} \neq 0$, one has $x_{\infty}=x_{r}$.

The consequence of this proposition is that if it is possible to find a robust controller that make $X_{\hat{p}}$ tend to $x_{r}$ then $x$ will tend to $x_{r}$ in spite of the external perturbation and the parameter uncertainties. As a result, the problem of disturbance rejection and robustness to model uncertainty for input delay systems is reduced to a problem of disturbance rejection and robustness to model uncertainty for delay-free systems. The only constraint that the controller should verify is that it is able to stabilize reduced system (14) which is possible when the uncertainties are not too large. Note that this limitation is related to the system and the controller rather than to the predictive scheme: even in the delay free case, it may be impossible to stabilize the system if parametric uncertainties are too large.

In this section the delay was supposed to be known and constant. In the next section, an approximated prediction and a delay estimator are going to be combined to deal with unknown and time-varying delays.

\section{DELAY ESTIMATOR AND PREDICTION FOR UNKNOWN AND TIME-VARYING DELAY}

In real applications, the delay is often time-varying because of network congestion for example. In addition, measuring the delay is quite difficult and usually only an approximation is available for control design. Consequently, an approximated prediction based on a delay estimation is presented in this section.

Consider the system

$$
\dot{x}(t)=A x(t)+B u(t-h(t))
$$

where the delay satisfies the following assumption

Assumption 5: The delay $h(t)$ is time-varying and unknown and there exist $h_{\min }, h_{\max }, \delta>0$ such that

$$
h_{\min } \leq h(t) \leq h_{\max }
$$

and

$$
|\dot{h}(t)| \leq \delta
$$

The exact prediction for time-varying delay is very difficult to compute since it requires a prediction of the delay [24], [25]. Assuming that the delay is slow-varying, a good approximation of the prediction is to use the same expression as in the constant delay case (4):

$$
x_{\hat{p}}(t)=e^{A \hat{h}} x(t)+\int_{t-\hat{h}(t)}^{t} e^{A(t-s)} B u(s) \mathrm{d} s
$$

but where the delay is substituted by its approximation $\hat{h}$. Since the delay usually cannot be measured, it is necessary to 
find a solution to estimate it. The delay is neither a parameter neither a state so standard techniques of identification and observation cannot be applied directly. Thereafter, an optimization method (similar to the one proposed in [26]) is presented to estimate a time-varying delay.

First, let us recall that the delay observability condition is:

Assumption 6 ([17]): For all $t \geq 0$,

$$
\dot{u}(t) \neq 0 \text {. }
$$

Condition (27) is natural because if the input is constant, the delay has no influence on the system so it cannot be observed. Note that Assumption 6 could appear restrictive because it means that the input should be strictly monotonic but in practice the notion of persistency ${ }^{3}$ given in [27] can be used to relax this condition. For a technical purpose, Assumption 6 can be substituted as follows

Assumption 7 ([26]): There exist time intervals $I$, sufficiently large, where the first derivative of $u$ is continuous and bounded: for all $t \in I$,

$$
|\dot{u}(t)|>\alpha
$$

with $\alpha>0$.

In addition, it is assumed that

Assumption 8: The delayed value of the input is known: the signal $u(t-h(t))$ is available.

Remark 2: In practical application, systems are able to save the input value received at instant $t$ and to send it back to the controller along with the measurement. The dashed line on Fig. 5 illustrates this process. The advantage of this method is to avoid the use of time stamped packets and clock synchronization.

This delay estimator is based on the minimization of the criterion

$$
J(\hat{h})=\|u(t-\hat{h}(t))-u(t-h(t))\|^{2}
$$

on $\left[h_{\min }, h_{\max }\right]$. Defining the dynamics of the delay estimation $\tau_{h}$ thanks to the gradient descent algorithm ${ }^{4}$, one has

$$
\dot{\hat{h}}(t)=-\left.\rho_{h}[u(t-\hat{h}(t))-u(t-h(t))] \frac{\partial u(t-\hat{h})}{\partial \hat{h}}\right|_{\hat{h}(t)}
$$

with $\rho_{h}>0$. Since

$$
\frac{\partial u(t-\hat{h})}{\partial \hat{h}}=-\frac{\partial u(t-\hat{h})}{\partial t},
$$

it results that

$$
\tau_{h}(t)=\rho_{h}[u(t-\hat{h}(t))-u(t-h(t))] \dot{u}(t-\hat{h}(t)) .
$$

Besides, to guarantee that $\hat{h}$ will stay in the interval $\left[h_{\min }, h_{\max }\right]$, a projection is used as follows:

$$
\dot{\hat{h}}=\operatorname{Proj}_{\left[h_{\text {min }}, h_{\text {max }}\right]}\left(\tau_{h}\right)
$$

\footnotetext{
${ }^{3}$ Roughly speaking, an input is said to be persistent for a system if it sufficiently rich to excite this system in order to estimate some parameters or to reconstruct its state.

${ }^{4}$ See the work of [28] for details about gradient descent algorithms.
}

with

$$
\operatorname{Proj}_{\left[h_{\text {min }}, h_{\text {max }}\right]}\left(\tau_{h}\right)= \begin{cases}0, & \text { for } \hat{h}=h_{\text {max }} \text { and } \tau_{h}>0 \\ 0, & \text { for } \hat{h}=h_{\text {min }} \text { and } \tau_{h}<0 \\ \tau_{h} & \text { else. }\end{cases}
$$

Assumption 9: The initial condition of the delay estimator satisfies $\hat{h}(0) \in\left[h_{\min }, h_{\max }\right]$.

If Assumption 9 is verified, then [29] guarantees that

$$
\forall t \geq 0, \quad \hat{h}(t) \in\left[h_{\min }, h_{\max }\right] .
$$

The following theorem states the convergence property of the above estimator.

Theorem 1: Let $I=\left[t_{0}, t\right]$ with $t_{0}>0$ be such that $t-$ $\hat{h}(t) \in I$ and $t-h(t) \in I$, if $|\dot{u}(s)|>\alpha$ for all $s \in I$, then the delay estimation error resulting from estimator (32)-(33) verifies

$$
\|e(t)\| \leq\left\|e\left(t_{0}\right)\right\| e^{-\rho_{h} \alpha^{2}\left(t-t_{0}\right)}+\frac{1}{\rho_{h} \alpha^{2}} \sup _{s \geq t_{0}}|\dot{h}(s)|
$$

for all $t \in I$ and with $e(t)=\hat{h}(t)-h(t)$.

Proof: For sake of clarity, the argument $t$ will be omitted when no confusion is possible. In [30, Chapter 4], it is proved that projection (34) retains all the properties that are established in the absence of projection that is why it is considered that

$$
\dot{\hat{h}}(t)=\rho_{h}[u(t-\hat{h}(t))-u(t-h(t))] \dot{u}(t-\hat{h}(t))
$$

in the sequel. Let

$$
V=\frac{1}{2}(\hat{h}-h)^{2}
$$

be a Lyapunov candidate function. Taking the time derivative of $V$ along the trajectories of (37) gives

$$
\dot{V}=\rho_{h}(\hat{h}-h)[u(t-\hat{h})-u(t-h)] \dot{u}(t-\hat{h})-(\hat{h}-h) \dot{h} .
$$

Reminding that from the mean value theorem there exists $\phi_{t} \in$ $[\min (t-h, t-\hat{h}), \max (t-h, t-\hat{h})]$ such that

$$
\dot{u}\left(\phi_{t}\right)=\frac{u(t-\hat{h})-u(t-h)}{h-\hat{h}},
$$

one gets

$$
\dot{V}=-\rho_{h}(\hat{h}-h)^{2} \dot{u}(t-\hat{h}) \dot{u}\left(\phi_{t}\right)-(\hat{h}-h) \dot{h} .
$$

Since $t-\hat{h}$ and $\phi_{t}$ are in the interval $I$, the terms $\dot{u}(t-\hat{h})$ and $\dot{u}\left(\phi_{t}\right)$ have the same sign. It follows that

$$
\dot{V} \leq-\rho_{h} \alpha^{2}(\hat{h}-h)^{2}-(\hat{h}-h) \dot{h}
$$

and then that

$$
\dot{V}(t) \leq-\rho_{h} \alpha^{2}\|\hat{h}-h\|^{2}+\|\hat{h}-h\| \sup _{s \leq t}|\dot{h}(s)| .
$$

Applying Lemma 9.4 in [31] leads to (36).

When the delay is constant, $\dot{h}=0$, the delay estimation error converges exponentially to zero. For a noisy signal, the alternative dynamics can be used

$$
\tau_{h}(t)=\frac{\rho_{h}}{T} \int_{t-T}^{t}[u(t-\hat{h}(t))-u(t-h(t))]^{T} \dot{u}(t-\hat{h}(t)) \mathrm{d} s
$$




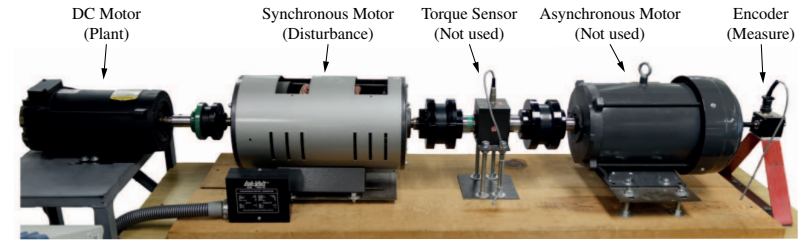

Fig. 1. Test Bench

where $T>0$ is the window's length that can be adjusted with respect to the noise level: the larger the noise is, the larger $T$ should be. Note that it is the filtered version of the dynamics defined in (32). The integral acts as a low pass filter whose time-constant is tuned by the parameter $T$. Similarly, if the input signal is fast varying, (44) can be normalized as follows

$$
\tau_{h}(t)=\frac{\rho_{h}}{T} \frac{I(t)}{\epsilon+|I(t)|}
$$

with $I(t)=\int_{t-T}^{t}[u(s-\hat{h}(s))-u(s-h(s))]^{T} \dot{u}(s-\hat{h}(s)) \mathrm{d} s$ and $\epsilon>0$. This normalization allows to make the convergence speed almost independent from the input dynamics. The dynamics of the estimators (44) and (45) are directly derived from (32) so the convergence can be derived from the proof of Theorem 1.

\section{EXPERIMENTAL VALIDATION}

\section{A. Setup presentation and model}

1) Experimental setup: the experimental setup is composed by a DC motor, a synchronous motor, a torque sensor, an asynchronous motor and an encoder. They are coupled by flexible joints. The plant to control is the DC motor and the synchronous motor has been used to introduce an external disturbance. The torque sensor and the asynchronous motor are not used in these experimentations. The control is computed thanks to a dSPACE board (DS1104). The optical encoder gives the angular position (1000 pulses per revolution) and the speed is derived from a Kalman filter. The dSPACE board uses the real-time model produced via Matlab/Simulink. The delay is artificially introduced in the loop by adding a delay block in the Simulink model. The armature voltage of the DC motor is controlled by a PWM signal generated by the dSPACE from the scaled input voltage $u(t)=\frac{v(t)}{v_{\max }}$ with $v(t)$ the equivalent continuous voltage and $v_{\max }=54 \mathrm{~V}$.

2) Model and control design: the transfer function of the DC motor with a retarded input $u$ is:

$$
\frac{\Omega(s)}{U(s)}=\frac{K}{1+s \tau} e^{-h s} .
$$

with $\Omega$ and $U$ the Laplace transforms of the angular velocity $\omega$ and the input voltage $u$ respectively. The steady-state gain $K$ and the time constant $\tau$ are known. The identified values of $K$ and $\tau$ are $K=894 \mathrm{~min}^{-1}$ and $\tau=1.10 \mathrm{~s}$. The transfer function $\frac{K}{1+s \tau}$ is a classical simplified model for DC motors where the inductance term can be neglected. The delay $h$ is not internal to the motor model but is supposed to be introduced by the control input, as for example, by the remote control over a network. The model is assumed to be linear and then does not take into account any nonlinear phenomenon such as dry friction. To apply above results, system (46) has to be turned into its state-space representation

$$
\dot{\omega}=a \omega+b u(t-h)
$$

with $a=-1 / \tau$ and $b=K / \tau$. Considering parameter uncertainties and external disturbance, system (47) becomes

$$
\dot{\omega}=(a+\Delta a) \omega+(b+\Delta b) u(t-h)+d
$$

with $a$ and $b$ known nominal terms, $\Delta a$ and $\Delta b$ unknown constant modeling errors and $d$ a constant unknown external disturbance.

Remark 3: Only the constant disturbance case is studied because, for practical reasons, it is not possible to apply a repeatable time-varying disturbance with the experimental setup at our disposal. Indeed, the external disturbance was created by connecting the synchronous motor (Fig. 1) to deliver a current into a resistance. The disturbance is then proportional to the value of the resistance but this latter can not be modified. Furthermore, the exact value of perturbation $d$ is not known but it is the same value for each experiment.

Remark 4: The model of the motor is very accurate so some additional uncertainties have been intentionally added to test the new prediction. However, the parameters of the DC motor cannot be modified easily so the uncertainties are added to the computation of the standard prediction as follows

$$
\omega_{\hat{p}}(t)=e^{(a+\Delta a) h} \omega(t)+\int_{t-h}^{t} e^{(a+\Delta a)(t-s)}(b+\Delta b) u(s) \mathrm{d} s .
$$

with $\Delta a=-0.2 a$ and $\Delta b=0.2 b$ ( $\pm 20 \%$ error).

The reference trajectory is given by $\omega_{r}$ so from the equilibrium equation $0=a \omega_{r}+b u_{r}$, one has $u_{r}=-\frac{a}{b} \omega_{r}$. From the expression of the standard prediction $\omega_{\hat{p}}$ in (49), the new prediction $W_{\hat{p}}$ reads as

$$
W_{\hat{p}}(t)=\omega_{\hat{p}}(t)+\omega(t)-\omega_{\hat{p}}(t-h) .
$$

Remark 5: The computation of $\omega_{\hat{p}}$ from (49) requires an integration. Usually, the integral has to be discretized in a finite number of points. However, this method can lead to instability for some systems [32]. Since the DC motor is open-loop stable, it is possible to compute the integral part of (49) without discretizing the integral, by using the structure given in [23]. Note that for open-loop unstable systems, a safe implementation of the prediction is given in [33].

The advantage of the predictive schemes is that it is possible to use any controllers available for delay-free systems and just "plug" the prediction $w_{\hat{p}}$ or $W_{\hat{p}}$ instead of the normal state $\omega$. To test and compare the schemes, a PI controller and a Super Twisting Algorithm ${ }^{5}$ (STA) will be designed. It is important to keep in mind that the objective is to compare both predictive schemes and to illustrate that they can be used with any kind of robust controllers. Consequently, no comparison between PI controller and STA will be drawn.

\footnotetext{
${ }^{5}$ Some first order sliding mode controllers have already been proposed to deal with input delay systems but with the standard prediction: see [34], [35] and [36].
} 
a) PI controller: the PI controller is defined by

$$
u(t)=u_{r}-k_{p} \chi(t)-k_{i} \int_{0}^{t} \chi(s) \mathrm{d} s
$$

with $\chi=\omega_{\hat{p}}-\omega_{r}$ for the standard scheme and $\chi=W_{\hat{p}}-\omega_{r}$ for the new predictive scheme. The gains $k_{p}$ and $k_{i}$ are $k_{p}=0.001$ and $k_{i}=0.002$.

b) Super Twisting Algorithm (STA): [37] the Super Twisting controller is a second order sliding mode controller. Its expression is given below:

$$
u(t)=u_{r}+\frac{1}{b}\left[-a \chi(t)-k_{1} \sqrt{|\chi(t)|} \operatorname{sign}(\chi(t))+\nu(t)\right]
$$

with the dynamics of $\nu$ governed by the following equation

$$
\dot{\nu}=-k_{2} \operatorname{sign}(\chi(t)) \text {. }
$$

with $\chi=\omega_{\hat{p}}-\omega_{r}$ for the standard scheme and $\chi=W_{\hat{p}}-$ $\omega_{r}$ for the new predictive scheme. The gains should satisfy $k_{2}>0$ and $k_{1}>\sqrt{2 k_{2}}$. Here, the following choice has been made $k_{1}=0.25$ and $k_{2}=0.025$. This controller does not require the acceleration values and achieves a robust finitetime convergence.

\section{B. Experimental results}

This section is divided into three subsections. First, the performances of the predictive control are studied with a known delay. Then, the delay estimator is tested. Finally, the combination of the predictive schemes and the delay estimation is illustrated.

1) Predictive control with a known delay: this part is not focused on controller performances but rather on the difference between the two predictive schemes. Both controllers (PI and STA) have been tuned on the delay-free system in order to reject the perturbation and the parameter uncertainties. The delay is chosen equal to $h=1 \mathrm{~s}$ in order to have the same order of magnitude for the delay and dynamics of the system (represented by $\tau=1.1 \mathrm{~s}$ ) which means that the effect of delay is not negligible. Some parameter uncertainties (given in Remark 4) affect the computation of the prediction and a perturbation $d$ is introduced by the synchronous motor between $50 \mathrm{~s}$ and $80 \mathrm{~s}$. The objective is to make $\omega$ track the reference velocity $\omega_{r}$ in spite of the delay $h$, the modeling errors $\Delta a, \Delta b$ and the external disturbance $d$.

The results for the constant and known delay case are shown on Fig. 2 and 3. On Fig. 2, PI controller (51) is considered. As it was required in Propositions 1 and 2, both predictions are able to track the reference $\omega_{r}$ in spite of the parameter uncertainties and the external disturbance $d$. However, only the motor controlled by the new predictive scheme guarantees the tracking of $\omega$ to $w_{r}$ (Fig. 2-Top) as stated in Proposition 2. On the contrary, in accordance to Proposition 1, the standard predictive scheme does not guarantee the asymptotic tracking of $\omega$ to $\omega_{r}$ (Fig. 2-Bottom). Roughly speaking, since the model is not accurate the standard prediction does not achieve to predict correctly the state of the system whereas the new prediction does (thanks to the correction term " $\omega(t)-\omega_{\hat{p}}(t-h)$ " in (50)).
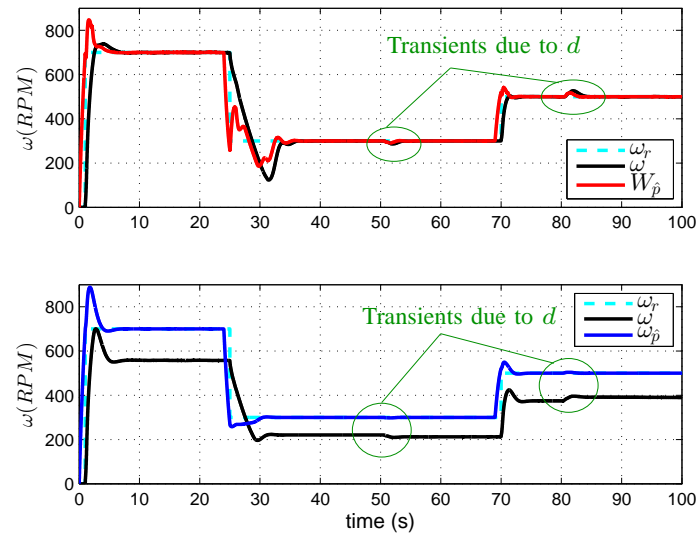

Fig. 2. Experimental results with PI controller and known and constant delay $h=1 \mathrm{~s}$, a constant disturbance $d$ between $50 \mathrm{~s}$ and $80 \mathrm{~s}$ and $\Delta a=-0.2 a$ and $\Delta b=0.2 b-$ Top: PI controller (51) with new prediction: $\chi=W_{\hat{p}}-\omega_{r}$ - Bottom: PI controller (51) with standard prediction: $\chi=\omega_{\hat{p}}-\omega_{r}$.
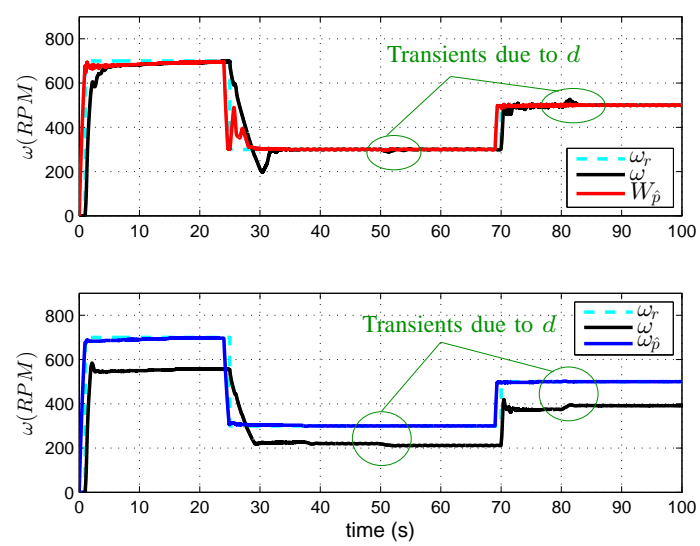

Fig. 3. Experimental results with STA controller and known and constant delay $h=1 \mathrm{~s}$, a constant disturbance $d$ between $50 \mathrm{~s}$ and $80 \mathrm{~s}$ and $\Delta a=$ $-0.2 a$ and $\Delta b=0.2 b-$ Top: STA controller (52) with new prediction: $\chi=W_{\hat{p}}-\omega_{r}-$ Bottom: STA controller (52) with standard prediction: $\chi=\omega_{\hat{p}}-\omega_{r}$.

Similarly, on Fig. 3, the STA controller guarantees the convergence of both predictions $\omega_{\hat{p}}$ and $W_{\hat{p}}$ to the reference $\omega_{r}$ in spite of the disturbance and the uncertainties. However, only the speed $\omega$ obtained from the new predictive scheme is able to track the reference $\omega_{r}$ (Fig. 3-Top). The velocity $\omega$ from the standard prediction scheme displays constant tracking errors (Fig. 3-Bottom). This validates once again Propositions 1 and 2 .

These experimental results confirm that the new predictive scheme associated to any kind of robust controllers provides better tracking performances than the standard predictive scheme.

2) Delay estimator performance: in this section, the performance of the delay estimator (33)-(45) are illustrated. The delay estimator parameters are $\rho_{h}=50, \epsilon=0.1$ and $T=200$ s. A PI controller is applied to stabilize the system around a piecewise constant trajectory. On Fig. 4, the velocity $\omega$ is not displayed because the objective is to illustrate the behavior of the delay estimator and not to study the trajectory tracking. Various phases are distinguishable. First, when the input saturates then $\dot{u}=0$ and the delay is unobservable. 

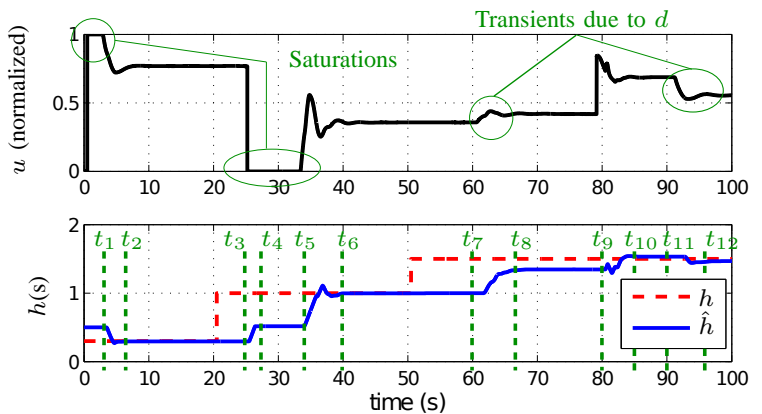

Fig. 4. Experimental results with PI controller and piecewise constant and unknown delay $h(t), \omega_{r}$ is piecewise constant, a constant disturbance $d$ between $60 \mathrm{~s}$ and $90 \mathrm{~s}-$ Top: control input - Bottom: exact delay $h(t)$ and estimated delay $\hat{h}(t)$.

This explains the two stages between $t=0$ and $t=t_{1}$ and between $t=t_{4}$ and $t=t_{5}$. Remark that the delay estimator does not diverge reaching this singularity; this is a crucial advantage over the work presented in [17]. Similarly, when $\omega$ has converged to $\omega_{r}$, the input becomes constant so $\dot{u}=0$. Consequently, the delay estimate cannot converged to the exact value: see for example between $t=t_{2}$ and $t=t_{3}$ and between $t=t_{8}$ and $t=t_{9}$. The delay estimator can only give a good estimation when the input varies. The origin of input variation can be the change of the reference such as between $t=t_{3}$ and $t=t_{4}$ and between $t=t_{9}$ and $t=t_{10}$ or it can be due to a disturbance such as between $t=t_{7}$ and $t=t_{8}$ and between $t=t_{11}$ and $t=t_{12} \mathrm{~s}$. This illustrates that the input should move sufficiently (to be persistent) in order to estimate accurately the delay. This constraint is inherent to the input delay and the quality of the estimation will always depend on the richness of the input signal. However, it is not very prejudicial when the objective is to achieve the stability of the closed loop system (which is possible even with an inexact delay estimation). Note that the same conclusion holds for a sufficiently slow time-varying delay as it will be shown in the next section.

3) Predictive control with the estimated delay: in this section, the delay estimator (33)-(45) is combined with predictions (49) and (50) by substituting the unknown real delay $h$ by its estimation $\hat{h}$. The whole scheme is shown on Fig. 5 . Note that the convergence proof of the prediction and the delay estimation have been given separately in Sections II and III. Intuitively, if the delay estimation is not accurate, the system is going to get away from the equilibrium point so the control is going to vary. Thus, Assumption 7 will be verified and the delay estimator will be able to get closer to the exact value and so on until the delay is sufficiently accurate to guarantee the convergence of the system. No theoretical proof of the stability of the closed-loop system is available since the result strongly depends on the choice of the controller. This issue will be considered in future developments. However, the efficiency of the combination of both techniques is illustrated thereafter by experimental results.

The results are shown on Fig. 6 and 7 for different delays $h(t)$. Note that the same parameter uncertainties and external disturbance as in Section IV-B1 are applied.

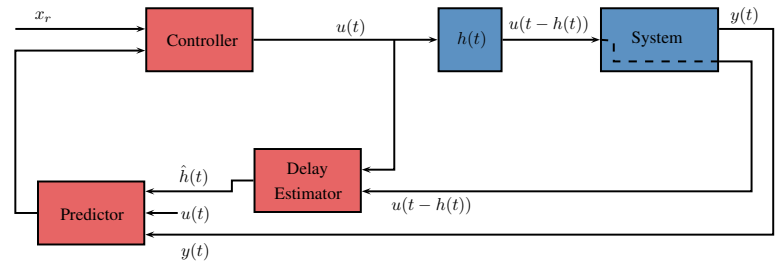

Fig. 5. Closed-loop scheme with the combination of the delay estimator and the predictor (see Remark 2 for the dashed line)
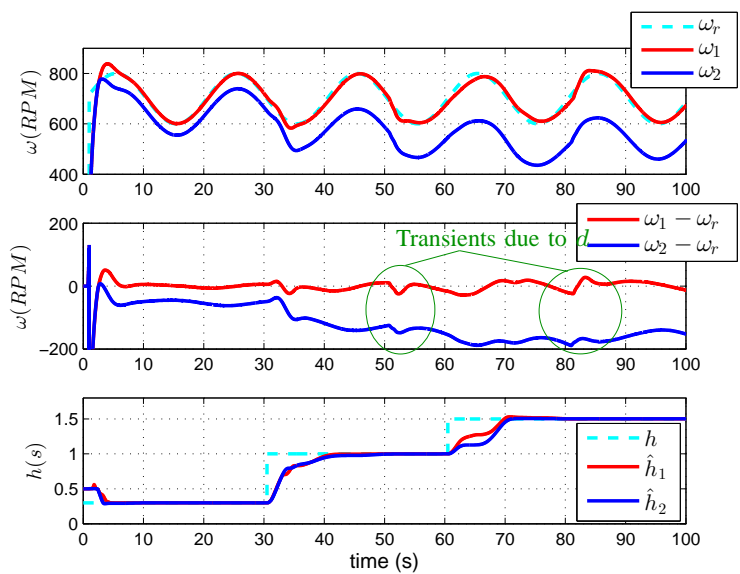

Fig. 6. Experimental results with PI controller and piecewise constant and unknown delay $h(t), \omega_{r}=700+100 \sin (0.3 t)$ RPM, a constant disturbance $d$ between $50 \mathrm{~s}$ and $80 \mathrm{~s}$ and $\Delta a=-0.2 a$ and $\Delta b=0.2 b$. The speed $\omega_{1}$ and the delay estimate $\hat{h}_{1}$ are obtained from the new predictive scheme whereas $\omega_{2}$ and $\hat{h}_{2}$ are obtained from the standard predictive scheme Top: velocity trajectories - Middle: tracking errors - Bottom: exact delay $h(t)$ and estimated delays.

On Fig. 6, one can see that the piecewise constant delay is correctly estimated for both predictive schemes. It can be noted that the delay estimation is not destabilized by the disturbance. As in the above section, only the new predictive scheme allows an effective tracking of the velocity reference in spite of the uncertainties and the external disturbance: $\omega_{1}$ correctly tracks $\omega_{r}$ whereas $\omega_{2}$ displays a large tracking error.

Fig. 7 shows that when the delay is time-varying, the estimation error converges in a ball around the exact delay as mentioned in Theorem 1. The small oscillations that can be observed on the delay estimates comes from the periodic cancellation of the input derivative. Indeed, when $\dot{u}$ gets closer to zero, the dynamics of the estimator tends to zero. However, the estimation is sufficiently accurate to guarantee an efficient tracking with the new predictive scheme. Like in the previous cases, the standard predictive scheme exhibits a large tracking error due to modeling errors $\Delta a, \Delta b$ and external disturbance $d$.

These two plots show that the new predictive scheme combined with the delay estimator (33)-(45) provide an efficient trajectory tracking in presence of time-varying and unknown delay and in spite of parameter uncertainties and external disturbance.

\section{CONClusion}

In this work, a recent predictive scheme is associated to a delay estimator. This combination is an efficient way to 

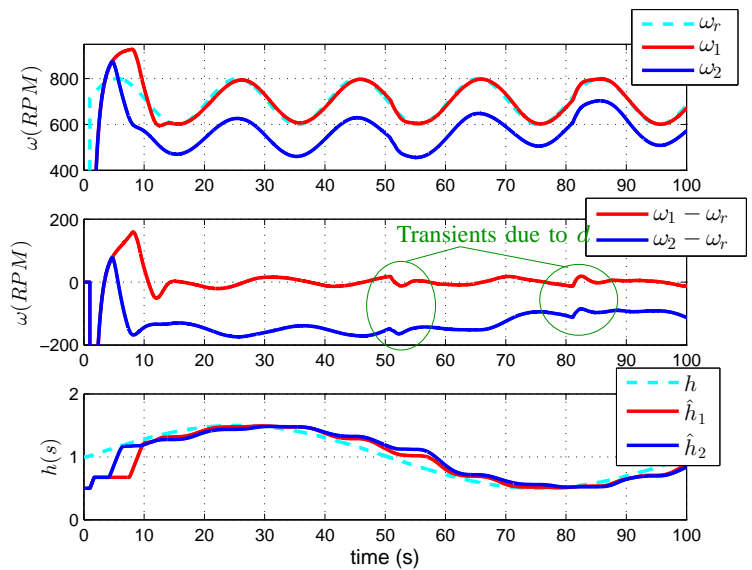

Fig. 7. Experimental results with PI controller and time-varying and unknown delay $h(t), \omega_{r}=700+100 \sin (0.3 t)$ RPM, a constant disturbance $d$ between $50 \mathrm{~s}$ and $80 \mathrm{~s}$ and $\Delta a=-0.2 a$ and $\Delta b=0.2 b$. The speed $\omega_{1}$ and the delay estimation $\hat{h}_{1}$ are obtained from the new predictive scheme whereas $\omega_{2}$ and $\hat{h}_{2}$ are obtained from the standard predictive scheme - Top: velocity trajectories - Middle: tracking errors - Bottom: exact delay $h(t)$ and estimated delays.

fix the standard drawbacks of predictive control; namely, the knowledge of the delay value and the sensitivity to parameter uncertainties and external disturbances. An extensive comparison with standard predictive techniques is made throughout the paper. The efficiency of the proposed scheme is validated experimentally on a DC motor. In some future works, it would be interesting to proof theoretically the convergence of the closed-loop system when the delay estimation is used in the prediction.

\section{REFERENCES}

[1] T. Vyhlídal, J.-F. Lafay, and R. Sipahi, Eds., Delay systems: From theory to numerics and applications, ser. Advances in Delays and Dynamics. Springer, 2014, vol. 1.

[2] O. J. M. Smith, "A controller to overcome dead time," ISA Journal, vol. 6 , pp. 28-33, 1959.

[3] A. Manitius and A. W. Olbrot, "Finite spectrum assignment problem for systems with delays," IEEE Trans. Autom. Control, vol. 24, no. 4, pp. 541-552, 1979.

[4] Z. Artstein, "Linear systems with delayed controls: A reduction," IEEE Trans. Autom. Control, vol. 27, no. 4, pp. 869-879, 1982.

[5] S.-I. Niculescu, Delay effects on stability: a robust control approach, ser. Lecture Notes in Control and Information Sciences. Springer, 2001, vol. 269.

[6] V. Léchappé, E. Moulay, F. Plestan, A. Glumineau, and A. Chriette, "New predictive scheme for the control of LTI systems with input delay and unknown disturbances," Automatica, vol. 52, no. 2, pp. 179-184, 2015.

[7] V. I. Utkin, "Sliding mode control design principles and applications to electric drives," IEEE Trans. Ind. Electron., vol. 40, no. 1, pp. 23-36, 1993.

[8] K. Matsuo, T. Miura, and T. Taniguchi, "Speed control of a DC motor system through delay time variant network," in SICE-ICASE International Joint Conference, 2006, pp. 399-404.

[9] R. Luck and A. Ray, "Experimental verification of a delay compensation algorithm for integrated communication and control systems," International Journal of Control, vol. 59, no. 6, pp. 1357-1372, 1994.

[10] Y. Tipsuwan and M.-Y. Chow, "Control methodologies in networked control systems," Control Engineering Practice, vol. 11, no. 10, pp. 1099-1111, 2003

[11] V. Léchappé, O. Salas, J. de León Morales, F. Plestan, E. Moulay, and A. Glumineau, "Predictive control of disturbed systems with input delay: experimental validation on a DC motor," in Time-Delay Workshop, Ann Arbor, USA, 2015.
[12] G.-P. Liu, Y. Xia, J. Chen, D. Rees, and W. Hu, "Networked predictive control of systems with random network delays in both forward and feedback channels," IEEE Trans. Ind. Electron., vol. 54, no. 3, pp. 1282 1297, 2007.

[13] H. Zhang, Y. Shi, and A. S. Mehr, "Robust static output feedback control and remote pid design for networked motor systems," IEEE Trans. Ind. Electron., vol. 58, no. 12, pp. 5396-5405, 2011.

[14] A.-A. Ahmadi, F. R. Salmasi, M. Noori-Manzar, and T. A. Najafabadi, "Speed sensorless and sensor-fault tolerant optimal pi regulator for networked DC motor system with unknown time-delay and packet dropout," IEEE Trans. Ind. Electron., vol. 61, no. 2, pp. 708-717, 2014.

[15] X.-M. Sun, D. Wu, G.-P. Liu, and W. Wang, "Input-to-state stability for networked predictive control with random delays in both feedback and forward channels," IEEE Trans. Ind. Electron., vol. 61, no. 7, pp. 3519-3526, 2014

[16] H. Li, M.-Y. Chow, and Z. Sun, "Optimal stabilizing gain selection for networked control systems with time delays and packet losses," IEEE Trans. Control Syst. Technol., vol. 17, no. 5, pp. 1154-1162, 2009.

[17] V. Léchappé, J. de León Morales, E. Moulay, F. Plestan, and A. Glumineau, "Delay and state observer for SISO LTI systems," in American Control Conference, Chicago, USA, 2015.

[18] A. O'Dwyer, "A survey of techniques for the estimation and compensation of processes with time delay," Dublin Institute of technology, Tech. Rep. AOD.00.03, 2000

[19] D. Bresch-Pietri and M. Krstic, "Adaptive trajectory tracking despite unknown input delay and plant parameters," Automatica, vol. 45, no. 9 , pp. 2074-2081, 2009.

[20] D. Bresch-Pietri and M. Krstic, "Delay-adaptive predictor feedback for systems with unknown long actuator delay," IEEE Trans. Autom Control, vol. 55, no. 9, pp. 2106-2112, 2010.

[21] J. Herrera and A. Ibeas, "On-line delay estimation for stable, unstable and integrating systems under step response," ISA Transactions, vol. 51, no. 3, pp. 351-361, 2012.

[22] D. L. Gaudette and D. E. Miller, "Stabilizing a SISO LTI plant with gain and delay margins as large as desired," IEEE Trans. Autom. Control, vol. 59, no. 9, pp. 2324-2339, 2014.

[23] K. Watanabe and M. Ito, "A process-model control for linear systems with delay," IEEE Trans. Autom. Control, vol. 26, no. 6, pp. 1261-1269, 1981.

[24] M. Krstic, Delay compensation for nonlinear, adaptive, and PDE systems, ser. Systems and Control: Fundations and Applications. Springer, 2009.

[25] M. T. Nihtila, "Adaptive control of a continuous-time system with timevarying input delay," Systems \& Control Letters, vol. 12, no. 4, pp. 357-364, 1989

[26] S. Diop, I. Kolmanovsky, P. E. Moraal, and M. van Nieuwstadt, "Preserving stability-performance when facing an unknown time-delay," Control Engineering Practice, vol. 9, no. 12, pp. 1319-1325, 2001.

[27] G. Besançon, G. Bornard, and H. Hammouri, "Observer synthesis for a class of nonlinear control systems," European Journal of Control, vol. 2, no. 3, pp. 176-192, 1996.

[28] D. Liberzon and R. Tempo, "Common Lyapunov functions and gradient algorithms," IEEE Trans. Autom. Control, vol. 49, no. 6, pp. 990-994, 2004.

[29] E. Lavretsky, T. E. Gibson, and A. M. Annaswamy, "Projection operator in adaptive systems," 2011, arXiv:1112.4232.

[30] P. A. Ioannou and J. Sun, Robust adaptive control, ser. Dover Books on Electrical Engineering. Dover Publication, 2012.

[31] H. K. Khalil, Nonlinear Systems, 3rd ed. Prentice Hall, 2002.

[32] V. Van Assche, M. Dambrine, J.-F. Lafay, and J.-P. Richard, "Some problems arising in the implementation of distributed-delay control laws," in Conference on Decision and Control, Phoenix, USA, 1999.

[33] S. Mondié and W. Michiels, "Finite spectrum assignment of unstable time-delay systems with a safe implementation," IEEE Trans. Autom. Control, vol. 48, pp. 2207-2212, 2003.

[34] Y.-H. Roh and J.-H. Oh, "Sliding mode control with uncertainty adaptation for uncertain input-delay systems," International Journal of Control, vol. 73 , no. 13, pp. 1255-1260, 2000.

[35] K. J. Hu, V. R. Basker, and O. D. Crisalle, "Sliding mode control of uncertain input-delay systems," in American Control Conference, Philadelphia, USA, 1998.

[36] S. A. Al-Shamali, O. D. Crisalle, and H. A. Latchman, "An approach to stabilize linear systems with state and input delay," in American Control Conference, Denver, USA, 2003.

[37] A. Levant, "Sliding order and sliding accuracy in sliding mode control," International Journal of Control, vol. 58, no. 6, pp. 1247-1263, 1993. 